\title{
Alterstice
}

Revue internationale de la recherche interculturelle

International Journal of Intercultural Research

Revista International de la Investigacion Intercultural

\section{Terrains de recherche au « prisme interculturel de la traduction »}

\section{Nilima Changkakoti, Myriam Gremion, Marie-Anne Broyon et Anahy Gajardo}

Volume 2, numéro 1, 2012

Stratégies de (re)médiation en situation plurilingue. Études de cas en contextes de recherche et d'éducation

URI : https://id.erudit.org/iderudit/1077552ar

DOI : https://doi.org/10.7202/1077552ar

Aller au sommaire du numéro

Éditeur(s)

Alterstice

ISSN

1923-919X (numérique)

Découvrir la revue

Citer cet article

Changkakoti, N., Gremion, M., Broyon, M.-A. \& Gajardo, A. (2012). Terrains de recherche au « prisme interculturel de la traduction ». Alterstice, 2(1), 41-54. https://doi.org/10.7202/1077552ar

\section{Résumé de l'article}

Cet article, dont le titre emprunte la formule de Ladmiral (1998), s'intéresse à la façon dont le chercheur entre en contact avec son terrain lorsque son objet de recherche implique un Autre enculturé dans d'autres façons de penser et de communiquer. Quatre chercheures, ayant effectué leurs recherches indépendamment, mettent en commun leurs réflexions, sur la base de quatre terrains différents (deux dans un ailleurs " proche ", deux dans un ailleurs " lointain »). Elles se posent la question de l'activation de la culture par le biais de la langue lorsque langues et cultures ne sont pas partagées par chercheur et répondants, en se centrant en particulier sur l'asymétrie des langues et des cultures et ce qu'elle peut induire lors du recueil et de l'analyse des données. La confrontation a posteriori des quatre terrains de recherche à la lumière de cette problématique pointe la nécessité d'explicitation à toutes les étapes de la recherche.
(C) Nilima Changkakoti, Myriam Gremion, Marie-Anne Broyon et Anahy Gajardo, 2012
Ce document est protégé par la loi sur le droit d'auteur. L'utilisation des services d'Érudit (y compris la reproduction) est assujettie à sa politique d'utilisation que vous pouvez consulter en ligne.

https://apropos.erudit.org/fr/usagers/politique-dutilisation/ 


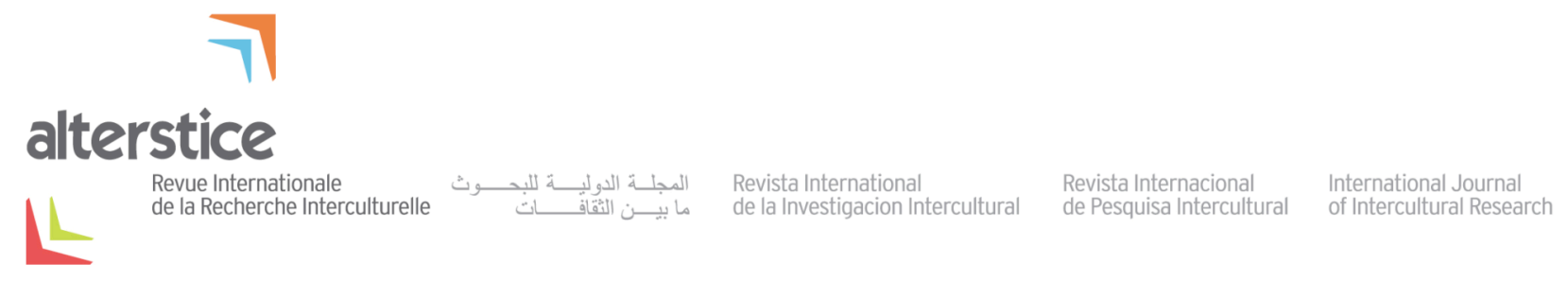

ARTICLE THÉMATIQUE

\title{
Terrains de recherche au " prisme interculturel de la traduction "
}

\author{
Nilima Changkakoti ${ }^{1}$, Myriam Gremion ${ }^{2}$, Marie-Anne Broyon ${ }^{3}$ et Anahy Gajardo ${ }^{4}$
}

\section{Résumé}

Cet article, dont le titre emprunte la formule de Ladmiral (1998), s'intéresse à la façon dont le chercheur entre en contact avec son terrain lorsque son objet de recherche implique un Autre enculturé dans d'autres façons de penser et de communiquer. Quatre chercheures, ayant effectué leurs recherches indépendamment, mettent en commun leurs réflexions, sur la base de quatre terrains différents (deux dans un ailleurs "proche ", deux dans un ailleurs " lointain »). Elles se posent la question de l'activation de la culture par le biais de la langue lorsque langues et cultures ne sont pas partagées par chercheur et répondants, en se centrant en particulier sur l'asymétrie des langues et des cultures et ce qu'elle peut induire lors du recueil et de l'analyse des données. La confrontation $a$ posteriori des quatre terrains de recherche à la lumière de cette problématique pointe la nécessité d'explicitation à toutes les étapes de la recherche.

\section{Rattachement des auteures}

${ }^{1}$ Université de Genève, Genève, Suisse

${ }^{2}$ Université de Genève et de Fribourg, Suisse

${ }^{3}$ Haute École Pédagogique du Valais (HEPVS), Suisse

${ }^{4}$ Fondation Éducation et Développement, Suisse

\section{Correspondance}

nilima.changkakoti@unige.ch

\section{Mots clés}

recherche interculturelle; traduction; questions méthodologiques

\section{Pour citer cet article :}

Changkakoti, N., Gremion, M., Broyon, M.-A. et Gajardo, A. (2012). Terrains de recherche au " prisme interculturel de la traduction ». Alterstice, 2(1), 41-54. 


\section{Introduction}

Dans cet article, dont le titre emprunte la formule de Ladmiral (1998), nous nous intéressons à l'enjeu de la langue dans le cadre de recherches où les interactions verbales constituent le mode de recueil de données privilégié. Notre réflexion se base sur quatre recherches menées indépendamment l'une de l'autre, avec des objectifs, des thèmes et des contextes différents (brièvement présentés dans le tableau 1 et sous le point 2), mais s'inscrivant toutes - à des degrés divers - « dans une approche sensible de terrains sensibles » (Fassin, 1999; Vulbeau, 2007), dans le sens d'une préoccupation à faire émerger des vécus, représentations, modes de pensée souvent interdits de parole parce que provenant de populations minoritaires en termes de rapports de pouvoir. Dans le cas présent, il s'agit de familles issues de la migration à Genève confrontées aux normes d'une culture scolaire parfois distante de ce qu'elles avaient pu connaître auparavant (tableau 1, terrains 1 et 2); d'un mode de scolarisation se référant à la tradition sanskrite comparé à "l'école à l'occidentale » en Inde (terrain 3), d'une population rurale au Chili travaillée par un processus de (re)construction identitaire autour de l'autochtonie en l'absence de la langue " originaire », perdue au profit de l'espagnol des ex-conquérants (terrain 4).

Le souci de faire entendre ces " voix cachées " (Murray et Wynne, 2001), que d'autres (Payet, Giuliani et Laforgue, 2008) appellent "la voix des acteurs faibles ", ou de questionner les positions universalistes (Mishrah et Dasen, 2007) nous a par ailleurs conduites à adopter des méthodologies privilégiant la construction, voire la coconstruction de sens entre chercheurs et participants.

L'aspect clinique impliqué par l'approche sensible, dans le sens d'un contact le plus direct possible avec les personnes participant à la recherche, impose par ailleurs une proximité linguistique, obtenue en l'occurrence par le recours à des traductrices ou interprètes ${ }^{1}$ et des assistantes de recherche ou permise par le fait que chercheure et participants partageaient a priori la même langue (dans la dernière situation analysée).

Toutefois, si le recours à un traducteur constitue la condition sine qua non de l'accès au terrain pour les trois premières situations analysées, ce choix n'est pas sans conséquences. II introduit d'autres paramètres dans l'interaction de recherche, qu'il rend de toute façon plus complexe, soit dans le sens d'un enrichissement, soit dans celui d'une complication accrue, source d'incompréhensions parfois insoupçonnées. Parler la langue de l'autre, comme c'est le cas pour le quatrième terrain, peut donc paraître un avantage indéniable par rapport aux situations nécessitant une médiation linguistique par le biais de la traduction. En effet, l'interaction directe facilite la communication et la compréhension entre les divers acteurs en jeu, permettant d'éviter les risques liés à l'interaction triangulaire induite par la présence d'un acteur-tiers. Cependant, l'atout que confère la maîtrise de la langue de son interlocuteur n'implique pas de facto l'intercompréhension et le partage d'un vocabulaire et d'un univers de significations communs. La langue peut sembler partagée, mais socioculturellement, les mêmes mots n'activent pas forcément les mêmes réalités (Goguikian Ratcliff et Changkakoti, 2004; Wadensjö, 1998). Par ailleurs, à l'intérieur d'une langue "standard", il peut exister une infinité de subtilités et variations modales exprimant des enjeux particuliers (identitaires, socioéconomiques, historiques). Ce type de situations peut donc entraîner des malentendus et des quiproquos d'autant plus difficiles à identifier et à démêler que l'interaction directe confère l'illusion de parler la même langue et partager le même univers de significations.

Ainsi, en contexte de communication interculturelle, quand il s'agit de traduire des langues et/ou des cultures, il ne s'agit jamais du simple passage d'une langue et d'une culture source à une langue et une culture cible. II faut obligatoirement tenir compte du prisme interculturel de la traduction (Ladmiral, 1998), et donc des différents éléments mentionnés ci-dessus.

Les heurs et malheurs du travail avec traducteur ont, jusqu'ici, plutôt été étudiés dans le contexte des contacts interculturels entre populations venues d'ailleurs et professionnels d'ici dans le domaine de la santé et de

\footnotetext{
${ }^{1}$ Comme ce n'est pas l'objet principal de l'article, nous ne traiterons pas ici de la question du terme adéquat pour désigner les traducteurs. Le terme de traducteur/translator étant fréquemment utilisé dans les articles de référence, c'est celui que nous utilisons ici.
} 
I'intervention psychosociale (Bischoff, Loutan et Stalder, 2001 ; Métraux, 2002 ; Searight et Searight, 2009 ; Weiss et Stucker, 1998). Hormis les textes classiques de l'anthropologie (Malinowsky, 1935; Philipps, 1960; Simon, 1988), la recherche interculturelle ne s'y intéresse que depuis peu (Bernhard, 2010; Buzelin, 2004; Londei, 2011; Murray et Wynne, 2001; Vatz-Laaroussi, 2003). Cette comparaison entre différents cas de figure de la recherche avec traducteurs ou non nous permettra donc à la fois de situer nos observations par rapport aux recherches sur la traduction-médiation dans le domaine de l'intervention et de nous inscrire dans cette nouvelle perspective de recherche.

Dans un premier temps nous tenterons de saisir certains de ces enjeux par une présentation des quatre terrains et des participants aux différentes recherches. Nous centrerons ensuite notre analyse sur la question de l'asymétrie des langues et des cultures partagées ou non, en termes de rapports de domination, statuts, etc. et sur ce qu'elle peut induire lors du recueil et de l'analyse des données dans les diverses situations discutées. Notre réflexion à quatre voix se fonde sur une analyse secondaire des entretiens recueillis, sur les notes ethnographiques et journaux de terrain précisant le contexte physique et social des interactions de recherche, sur les ressentis des chercheures sur le moment ainsi que sur une autoréflexion a posteriori. Il s'agit plus d'explorer divers cas de figure que d'effectuer une comparaison systématique inter-terrains.

\section{Présentation des terrains et acteurs des quatre recherches}

Comme cela a été mentionné en introduction, deux des terrains analysés se situent dans un ailleurs proche: familles migrantes en Suisse. Les deux autres dans un ailleurs lointain: élèves dans deux systèmes scolaires différents dans une même ville indienne, et une communauté autochtone au nord du Chili, en contexte rural. Le tableau ci-dessous récapitule les thèmes de recherche, les lieux où les données ont été recueillies, les langues en jeu dans les interactions de recherche et les approches méthodologiques mises en œuvre.

Tableau 1 : Terrains et méthodologies de recherche

\begin{tabular}{|l|l|l|l|}
\hline \multicolumn{1}{|c|}{ Thème de recherche } & \multicolumn{1}{|c|}{ Lieux } & Langues en jeu & \multicolumn{1}{c|}{ Méthodologie } \\
\hline $\begin{array}{l}\text { 1. Vécu de parents migrants lors de l'entrée } \\
\text { en institution scolaire (ordinaire ou } \\
\text { spécialisée) de leur enfant en situation de } \\
\text { handicap (2008 - en cours). }\end{array}$ & $\begin{array}{l}\text { Genève, } \\
\text { domicile des } \\
\text { familles }\end{array}$ & $\begin{array}{l}\text { Français } \\
\text { Tamoul, Panjabi }\end{array}$ & $\begin{array}{l}\text { Compréhensive, longitudinale, entretiens } \\
\text { semi-dirigés auprès de parents (père et mère } \\
\text { ou mère seule) à trois moments avec } \\
\text { intervalles de } 6 \text { mois. }\end{array}$ \\
\hline $\begin{array}{l}\text { 2. Impact de la scolarisation de l'aîné sur la } \\
\text { dynamique d'acculturation de familles } \\
\text { migrantes vivant à Genève } \\
\text { (2003- 2006). }\end{array}$ & Genève & $\begin{array}{l}\text { Français } \\
\text { Albanais }\end{array}$ & $\begin{array}{l}\text { Compréhensive, entretiens semi-dirigés } \\
\text { auprès de familles (parent(s) seul(s), } \\
\text { parents+enfants; aînés seuls, entretiens } \\
\text { collectifs femmes). }\end{array}$ \\
\hline $\begin{array}{l}\text { 3. Développement de la métacognition chez } \\
\text { des élèves d'écoles hindi et sanskrites } \\
\text { (2002- 2009). }\end{array}$ & Inde (Bénarès) & $\begin{array}{l}\text { Hindi Anglais } \\
\text { Français }\end{array}$ & $\begin{array}{l}\text { Mixte : Tâches métacognitives (épreuves } \\
\text { spatiales), questionnaire pour mesurer la } \\
\text { métacognition, observations et entretiens. }\end{array}$ \\
\hline $\begin{array}{l}\text { 4. Processus de reconstruction identitaire } \\
\text { des Diaguita, une communauté autochtone } \\
\text { hispanophone du nord du Chili ayant perdu } \\
\text { l'usage de sa langue « originaire » } \\
\text { (2006 -2012, analyse en cours). }\end{array}$ & $\begin{array}{l}\text { Chili (Province } \\
\text { d'Atacama) }\end{array}$ & $\begin{array}{l}\text { Espagnol } \\
\text { Français }\end{array}$ & $\begin{array}{l}\text { Ethnographique : entretiens semi-directifs et } \\
\text { non directifs, observation directe, observation } \\
\text { participante, séjours de longue durée, } \\
\text { photographies, films. }\end{array}$ \\
\hline
\end{tabular}

Dans une démarche similaire, une chercheure canadienne d'origine salvadorienne (Bernhard, 2010) se pose la question de la validité des données recueillies lorsque l'écart entre mandats de recherche (explicites ou implicites) et valeurs des participants et/ou des chercheurs entraîne des conflits identitaires, voire empêche l'accès aux données. Elle préconise de prêter plus d'attention à la formation professionnelle des chercheurs et à leur propre 
acculturation", comme "d'importants marqueurs dont il faut tenir compte dans le processus de recherche " (p. 30). Nous présentons donc ici nos enculturations personnelles et professionnelles ${ }^{3}$ comme pouvant contribuer à la création de ce prisme interculturel de la traduction de langues et de cultures (voir aussi sur ce thème Pirinoli, 2004).

Toutes les chercheures travaillent en sciences de l'éducation mais bénéficient d'autres ancrages disciplinaires comme la psychologie et l'ethnologie ${ }^{4}$. Leur langue d'interaction quotidienne et de travail est le français, mais chacune d'elles a d'autres connaissances linguistiques et l'une d'elles est bilingue (français - espagnol).

\section{Premier terrain}

La chercheure intervenant sur le premier terrain a une formation initiale en psychologie et effectue ses enseignements et ses recherches en sciences de l'éducation. Préalablement à cette étude, elle a suivi une formation sur la collaboration avec des interprètes. Les deux interprètes participant à la récolte des données ont des parcours de formation différents. La première a suivi une formation initiale en gestion et fonctionne actuellement comme interprète communautaire ${ }^{5}$ (tamoul-français) à la suite d'une formation de 180 heures dispensée par une association très active auprès des communautés immigrées, notamment dans le domaine de la santé mentale (pour en savoir plus: Fleury et Fierro, 2009; Métraux, 2002; Métraux et Alvir, 1995). De formation initiale diététicienne, la deuxième travaille comme interprète communautaire (panjabi-français) pour la CroixRouge genevoise (pour en savoir plus : Joss, 2008). Les personnes interrogées sont des parents migrants ayant deux jeunes enfants, dont un en situation de handicap. Les mères ont suivi l'école obligatoire au pays, sont ici femmes au foyer et parlent et comprennent peu le français. Les pères ont la même éducation de base, travaillent ici comme ouvrier ou restaurateur, l'un d'eux étant à l'assurance invalidité - ils comprennent tous assez bien le français.

\section{Deuxième terrain}

Pour la deuxième recherche, la personne ayant mené les entretiens analysés ici (qui constituent un sous-ensemble de la recherche complète, soit 8 entretiens sur 40, voir Perregaux, Changkakoti, Gremion et Hutter, 2010) est chercheure en éducation et psychothérapeute et fonctionne aussi dans une consultation d'ethnopsychiatrie. Elle est née en Suisse de parents issus de la migration. L'interprète, arrivée comme jeune adulte en Suisse venant du Kosovo a travaillé comme collaboratrice sociale dans une association communautaire, elle travaille comme interprète pour sa communauté, également pour des Bosniaques et parle français, albanais et bosniaque (ainsi que le croate et le serbe, langues proches). La chercheure et l'interprète ont déjà travaillé ensemble dans le domaine de la psychothérapie et l'interprète a joué le rôle d'intermédiaire pour solliciter la participation des familles à la recherche. Les familles sont originaires de Kosove, parlent albanais, le français dans certains cas, et ont souvent d'autres connaissances linguistiques acquises dans la migration, comme le suisse-allemand, ou au pays, comme le turc, le russe et le serbe. Les pères se débrouillent généralement mieux en français que les mères, sauf dans un cas où c'est l'inverse et une mère seule avec qui l'entretien a eu lieu directement en français. Les formations des pères vont de l'école obligatoire à l'université, celles des mères aussi, mais les femmes sont plus nombreuses à n'avoir pas eu l'opportunité d'aller au-delà de l'éducation de base. Les emplois occupés par les hommes sont généralement non qualifiés, indépendamment des formations, à une exception près (journaliste). La situation est similaire pour les femmes, là aussi à une exception près, et elles sont par ailleurs plus nombreuses à ne pas travailler en dehors du foyer.

\footnotetext{
${ }^{2}$ On entend ici l'intégration dans la société d'accueil des chercheurs issus eux-mêmes de la migration.

${ }^{3}$ On entend ici l'approprication inconsciente par les chercheurs de valeurs, idées, normes, etc. de leurs groupes d'appartenances personnelle et professionnelle. Pour un exemple, voir Hutter, Gremion, Borruat, Ogay, Zharkova Fattore et Gakuba (2008).

${ }^{4}$ La deuxième recherche a par ailleurs été menée par une équipe mixte : sciences de l'éducation, sociolinguistique, psychologie.

${ }^{5} \mathrm{~L}^{\prime}$ interprète communautaire fonctionne en tenant compte des contextes socioculturels des professionnels et des migrants qui le mandatent et recourt pour cela à sa double connaissance des langues et des cultures, ainsi qu'à sa propre expérience de la migration (www.inter-pret.ch).
} 


\section{Troisième terrain}

En ce qui concerne la troisième recherche, la chercheure travaille dans la formation des enseignants et la recherche en sciences de l'éducation après un long parcours professionnel dans le secteur privé (multinationales). Deux traductrices assistantes de recherche ont collaboré à l'étude. L'une, en possession d'un doctorat en psychologie cognitive, fonctionnait comme responsable du groupe de recherche et l'autre, enseignante et doctorante en sciences de l'éducation après des études internationales (Inde, Suisse et États-Unis), était chargée de la vérification des traductions (entretiens, résolutions de tâches métacognitives filmées, documents écrits). Les autres participants comprenaient des enseignants et directeurs d'établissements de diverses formations universitaires et les élèves fréquentant ces établissements (deux écoles sanskrites, une de filles et une de garçons, et une école hindi mixte) (Broyon, 2004). Mise à part une assistante musulmane, toutes les assistantes étaient hindouistes mais de castes diverses (seules la responsable du groupe et la vérificatrice étaient brahmanes).

\section{Quatrième terrain}

Enfin, la chercheure impliquée sur le quatrième terrain bénéficie d'une formation en anthropologie sociale et en sciences de l'éducation. Sa langue de communication scientifique est le français. Originaire du Chili, fille de parents natifs d'une région proche de son terrain, elle a cependant été socialisée et scolarisée en Suisse depuis sa plus tendre enfance en raison de l'exil politique de sa famille. Elle n'a donc connu physiquement le Chili que tardivement, notamment dans le cadre de ses recherches, et n'a appris l'espagnol qu'oralement, en exil, dans le contexte familial et communautaire. La langue des échanges sur le terrain est l'espagnol, sans intermédiaire. Les participants à la recherche sont des habitants de la région du Huasco-Alto (province d'Atacama,) petits agriculteurs et/ou éleveurs semi-nomades, peu ou pas scolarisés et s'auto-identifiant comme des " Diaguita », un ethnonyme qui jusqu'à la moitié des années 1990 désignait un peuple préhispanique de la région considéré comme disparu peu après la colonisation espagnole (voir Gajardo, 2009). Reconnus légalement en 2006 comme une ethnie autochtone, les Diaguita intensifient dès lors leurs efforts pour " restaurer » leur identité autochtone alors que leur langue, le kakán, n'est plus parlée et qu'il n'en existe aucune trace écrite. Le castillan parlé par les habitants de la région du Huasco-Alto est toutefois teinté de mots d'origines autochtones, dont les locuteurs méconnaissent souvent la provenance.

Les terrains présentés ici sont donc divers, mais, comme nous cela a été mentionné plus haut, traversés par des interrogations épistémologiques et méthodologiques communes, dont celle de l'incidence de l'asymétrie des langues et des cultures.

\section{Comment rester " sensibles " en situation d'asymétrie des langues et des cultures?}

\section{Écarts socioculturels, rapports de pouvoir et relations de confiance}

L'asymétrie principale présente dans les quatre terrains est celle qui se dessine entre chercheurs et répondants. Même si ces derniers pourraient être désignés comme des "co-chercheurs" dans la mesure où une coconstruction de sens est privilégiée, ce ne sont en général pas eux qui ont pris l'initiative de la rencontre. Par ailleurs, ils sont fragilisés par leur posture d' «acteurs faibles » induite par la méconnaissance d'un système, la présence d'une déficience chez leur enfant, l'absence d'un statut légal ou le vécu d'un statut précaire (terrains 1, 2 et 4).

À cela s'ajoute que les chercheures sont issues du milieu universitaire et que cette appartenance peut, qu'elles le veuillent ou non, influencer par moments l'interaction. II faut dire que l'identité attribuée au chercheur en début de terrain n'est pas nécessairement explicitée et maîtrisée (Sirna, 2009). Comme le montre l'exemple fameux de l'anthropologue Jeanne Favret-Saada enquêtant sur la sorcellerie dans le bocage normand, le chercheur est généralement absent des représentations des "enquêtés » et se trouve donc, souvent à son insu, placé dans l'une des positions prévues par la «situation d'énonciation »(Benveniste, 1966), en l'occurrence, pour Favret-Saada (1977, 2009), l'anthropologue était située soit du côté des sorciers, soit du côté des victimes de mauvais sorts.

L'effet est peut-être moins massif dans le cas présent, mais les chercheures (terrains 1 et 2) ont été perçues par moments comme des représentantes des institutions du pays d'accueil ou des représentantes de l'école, à qui il 
faut plaire ou résister. Sur le terrain 3, le monde statistiquement majoritaire de l'Inde (majority world, Dasen et Akkari, 2008) se trouve confronté aux idéologies dominantes du monde occidental, dont la chercheure venue du Nord ne se veut justement pas la représentante, mais qui ont pu dans une certaine mesure être intériorisées par certains participants locaux à la recherche (enseignants et enseignantes, directeurs et directrices, assistants et assistantes de recherche ayant étudié à l'étranger) et ont fait à ce titre partie du transfert sur la chercheues responsable de la recherche. Dans le même sens, dans le Huasco-Alto (terrain 4), la chercheure était parfois considérée comme une compatriote (même nationalité) et d'autres fois comme une étrangère, occidentale, nondiaguita, parfois comme une anthropologue pouvant contribuer à informer le processus de reconstruction identitaire à l'œuvre (apport) ou comme pouvant spolier la culture diaguita (menace), parfois comme un agent de la compagnie minière ou du gouvernement menaçant le territoire de la communauté ou comme une actrice pouvant soutenir la lutte pour sa défense et sa préservation.

L'explicitation de la démarche et de la volonté de donner voix à des discours, pratiques et représentations méconnues devrait permettre l'établissement d'une relation de confiance et y contribue certainement, mais elle n'empêche pas les projections et les chercheurs rencontrent parfois des surprises. Ainsi, lors d'un entretien avec une famille de Kosove (terrain 2), un malentendu se produit autour de la notion de changement ${ }^{6}$. La question posée concernait dans l'esprit de la chercheure à la façon dont le répondant percevait son propre parcours et la nature des éventuels changements relevés, alors que pour le père de famille, interlocuteur principal de l'entretien, " changement " renvoyait apparemment au progrès, la Suisse se présentant comme un pays de progrès face au Kosovo, pays arriéré.

Père : Mais je veux revenir à la question que vous avez posée ${ }^{7}$, ça m'a provoqué. Comment vous savez qu'on a changé, c'est quoi les critères, vous êtes allée au Kosovo? Vous savez comment c'est là-bas? On a complètement changé ici, y a pas de courant là-bas...?

Chercheure: Non, je ne sais pas, je ne connais pas le Kosovo, et je n'aurais pas la prétention de dire si vous avez changé ou pas, je ne vous connais pas et je ne connais pas le Kosovo. Ce qui m'intéressait c'est si vous, vous aviez l'impression d'avoir changé, ou non, depuis que vous vivez ici. C'est ce que vous pensez qui m'intéresse.

Père: Ah, j'avais mal compris. (Réfléchit un moment). À la vérité, je suis ici depuis (presque 20 ans), je suis toujours pas intégré... et je suis intégré. J'ai un pied ici et un pied là-bas.

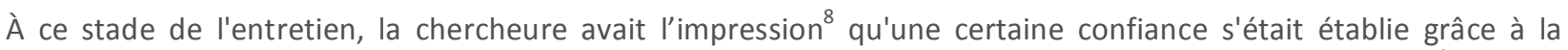
présence de la traductrice (connue de la famille), grâce à des échanges du « monde vécu » (Leanza, 2005) entre la traductrice et le père notamment sur le travail de médiateurs que tous deux ont effectué à un moment donné pour leur communauté, grâce à des échanges sur la langue où la chercheure a pu «montrer patte blanche » en mettant en jeu ses quelques connaissances linguistiques. On peut toutefois faire l'hypothèse que le mot changement comporte pour le père des connotations de jugement de valeur et provoque une réaction défensive ${ }^{10}$. Les rapports de force sont alors convoqués: la chercheure est placée dans une position de représentante d'une société d'accueil... peu accueillante et le père réagit à une pression à l'assimilation. Ce genre de projections sur les chercheurs peut aussi induire un discours de conformité sociale ou militant (rester dans le général, ne pas entrer dans le vécu), ce que nous avons pu vérifier dans les recherches présentées ici. La proximité ressentie n'est donc pas toujours partagée.

\section{Asymétrie des langues}

La langue est un marqueur identitaire (Billiez, 1985) fort et est donc tributaire des asymétries évoquées ci-dessus. Elle témoigne des appartenances socio-culturelles et de leurs ambivalences. En contexte de migration l'usage des

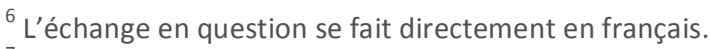

${ }^{7}$ Avez-vous l'impression d'avoir changé depuis que vous êtes en Suisse? (notre traduction)

${ }^{8}$ Ressenti de la chercheure, notes de terrain.

${ }^{9}$ A savoir ici, des échanges ne relevant pas directement de la thématique de l'entretien, initiés par le répondant et la traductrice.

${ }^{10}$ Il faut mentioner ici que le père était enseignant dans son pays.
} 
langues rend ainsi compte du processus d'acculturation : de l'épreuve de la conquête de la langue du pays d'accueil (Deprez, 2002), à la transmission de la langue (ou des langues) d'origine, en passant par la constitution de répertoires linguistiques pluriels. Dans une société pluriculturelle, toutes les langues présentes ne sont pas également représentées dans l'espace public et n'ont pas le même statut, les langues de la migration en particulier étant dévalorisées. Le recours à un traducteur, au-delà de l'objectif zéro de la compréhension, peut alors être pensé comme un acte symbolique de reconnaissance de la langue de l'autre. Les stigmatisations subies et intériorisées n'en disparaissent pas pour autant. Même s'il y a traduction, l'entretien part du français et y retourne. Comment se négocie par le biais de la traduction ce passage délicat d'une langue minoritaire à la langue officielle? Dans les entretiens familiaux du terrain 2, nous avons pu constater un passage de langue modulé par le rôle de I'individu dans l'acculturation du groupe familial (Changkakoti, Gremion et Hutter, 2009). En effet, les attitudes ne sont pas les mêmes selon les différents membres de la famille, ils n'ont pas la même maîtrise de la langue véhiculaire, et n'ont pas le même rapport aux langues en présence. Ainsi l'exemple d'un jeune témoignant d'un processus de "déni de langue " parce que sa langue maternelle est stigmatisée dans la société d'adoption : il dit en français qu'il ne veut pas prendre de cours d'espagnol "parce qu'il y a que des pauvres qui la parlent ", sa mère disant à un autre moment, en espagnol, qu'il lit et écrit dans sa langue maternelle.

Pour que la reconnaissance de l'autre reste dynamique, il faut sans doute une certaine flexibilité dans le passage des langues: à savoir que la communication ne soit pas uniquement activée à partir de la position du chercheur, admettre qu'il y ait des échanges parallèles dans une langue ou l'autre même si le chercheur n'y a pas immédiatement accès dans la mesure où, du fait de la traduction, il existe un délai entre émission et compréhension du message, accepter aussi qu'il y ait des moments d'échanges balbutiants dans la langue véhiculaire si le mouvement part du répondant, qu'il y ait alternance codique ou bricolage en recourant aux deux langues ou à des emprunts.

Cette flexibilité peut cependant difficilement exister d'emblée, le délai entre émission et compréhension du message caractéristique de la communication médiatisée est en effet souvent évoqué par les professionnels qui y recourent comme un frein à la "bonne " communication (Cohen-Emerique, 2011; Leanza, 2011). Dans les recherches présentées ici (terrains 1, 2 et 3), le rythme de l'entretien médiatisé permet au chercheur d'être plus attentif au langage non verbal, aux réactions des participants dont ce n'est pas le tour de parole lorsqu'il s'agit d'un entretien collectif. La compréhension différée peut cependant aussi interférer avec l'empathie, déposséder le chercheur d'une partie des clefs de l'entretien et remettre ainsi partiellement en question la démarche compréhensive. Lors d'un entretien mené autour de la scolarisation d'un enfant handicapé (terrain 1), une maman sri-lankaise se met ainsi à pleurer au tout début de l'entretien, alors qu'il s'agit d'une question "banale » de présentation. La chercheure se sent en empathie avec cette femme, mais sans vraiment comprendre pourquoi, se demande ce qu'il se passe, ce qui a pu causer cette émotion, s'interroge sur la suite de l'entretien, sur l'attitude à adopter. Elle se sent dans une position inconfortable et le temps d'attente de la traduction qui lui permettrait de décoder la situation lui paraît long ${ }^{11}$. L'interaction se construit alors comme un double dialogue qui interroge sur la construction de la relation entre chercheur et répondant et sur le degré de profondeur des propos échangés.

Au-delà de la déqualification des langues de la migration et de la compréhension différée, la question de l'asymétrie des langues se pose aussi au sein d'une même société. Le terrain de Bénarès (terrain 3) en est une bonne illustration. La référence linguistique (et culturelle) des deux types d'école n'est pas la même. Dans un cas, la langue d'enseignement est le hindi (une des langues officielles de I'Inde et langue véhiculaire locale), et la pédagogie s'inscrit dans l'héritage de l'école coloniale qui a fini par aboutir à une forme scolaire de type occidental uniformisée au niveau international. Dans l'autre cas, certains enseignements se font dans la langue sacrée, le sanskrit, devenue langue de communication, et la pédagogie s'inspire de l'éducation traditionnelle sanskrite (Broyon, 2004). En outre, la communication entre la chercheure principale venue de Suisse et les assistantes s'effectue en anglais, qui est une des langues officielles de l'Inde, la langue des ex-colons et l'une des langues scientifiques des chercheures.

\footnotetext{
${ }^{11}$ Ressenti de la chercheure, notes de terrain.
} 


\section{L'illusion de la langue partagée}

La langue est considérée comme un vecteur important de la culture, ce qui peut entraîner la double illusion que si I'on partage la langue, on partage aussi la culture et que si l'on partage la langue on se comprend. C'est faire fi des variétés modales à l'intérieur d'une langue dite standard, fi du marquage social de la langue (ethnographie de la communication, Blanchet, 2000; Gumperz, 1989). L'exemple suivant, rencontré sur le terrain diaguita (terrain 4), montre comment l'implicite de la langue partagée crée de l'incompréhension voire de la méfiance lorsque la chercheure, hispanophone d'origine chilienne, demande la signification d'un mot régional qu'elle ne connaît pas.

Femme diaguita : Mi abuelita [...] tenía una ñotuna en su patio, me acuerdo que molían el trigo las mujeres todas juntas y los niños nos alegrabamos porque eran momentos muy bonitos, y en la noche, se contaban historias, y sí se hablaba del Yastay [...]

Chercheure : La ñotu...? Me puede explicar de qué se trata?

Femme diaguita : La ñotuna.

Chercheure : Me puede explicar que es la ñotuna? Es un objeto, un...? No entiendo...

Femme diaguita : Es chilena o no? Habla español o qué...? Porqué me pide de explicar algo que usted sabe ya? ${ }^{12}$

La question de l'espagnol revêt par ailleurs une importance particulière pour les Diaguita. Ils revendiquent le statut de peuple autochtone en l'absence de ce marqueur identitaire considéré comme primordial qu'est la langue autochtone, ce qui entache leur revendication d'une suspicion d'inauthenticité. La langue disparue devient alors un instrument de lutte politique et ethnoculturelle notamment autour de la question de la transmission (Lavanchy et Gajardo, 2008). Dans une certaine mesure, la démarche de l'école sanskrite des filles à Bénarès s'inscrit aussi dans une revendication politique et ethnoculturelle ${ }^{13}$, où la langue sacrée rentre dans la sphère du temporel.

Les exemples cités montrent bien que la compréhension implique plus qu'un simple passage linguistique. La proximité linguistique est nécessaire mais elle n'est pas forcément suffisante pour accéder de façon fiable aux "voix cachées": malentendus, méfiance, implicites présumés partagés parasitent la communication entre chercheurs ou traducteurs et répondants parlant en apparence la même langue. II faut à nouveau tenir compte des différences socioculturelles incarnées dans la langue, des parlers régionaux, des mots à connotations différentes, des réalités différentes activées par un même mot.

\section{Postures du traducteur}

Quelle place donner alors au traducteur? Dans le domaine de l'intervention psychosociale avec traducteur, la littérature a pu relever différents rôles attribués au traducteur allant du modèle de la boîte noire (Jalbert, 1998) au rôle de co-thérapeute (Métraux et Alvir, 1995), en passant par les rôles d'avocat, de professionnel bilingue, de référent culturel et médiateur culturel (Kaufert et Putsch, 1997; Leanza, 2005). Avec un même objectif avoué d'établir une relation de confiance, les pratiques couvrent le spectre des rôles évoqués ci-dessus.

L'invisibilité du traducteur peut être vue comme une condition à une empathie la plus directe possible, sa présence étant souvent perçue comme un obstacle dans un modèle dyadique assez prégnant de la relation de confiance (Molina, 2001; Weiss et Stucker, 1998). En fonctionnant le plus possible comme une machine, donc de façon neutre, les effets de filtre et d'interprétation devraient être minimisés (Apostolou, 2009; Graber et Girod, 2001; Molina, 2001). La métaphore du traducteur comme un simple conduit reste assez fréquente, y compris chez les

\footnotetext{
${ }^{12}$ Ma grand-mère (diminutif affectueux) avait une ñotuna dans sa cour, je me souviens que les femmes se mettaient toutes ensemble pour moudre le blé et que nous les enfants on se réjouissait parce que c'était des bons moments, et le soir on racontait des histoires et on parlait du Yastay. / La ñotu...? Vous pouvez m'expliquer de quoi il s'agit? / La ñotuna. / Vous pouvez m'expliquer ce que c'est la ñotuna, un objet...un...? Je ne comprends. / Vous êtes chilienne ou pas? Vous parlez espagnol ou quoi? Pourquoi vous me demandez d'expliquer quelque chose que vous savez déjà? [notre traduction]

${ }^{13}$ Proches du fondamentalisme religieux hindouiste, certains enseignements (arts martiaux, slogans scandés pendant l'entraînement) adoptent un ton assez guerrier.
} 
traducteurs eux-mêmes (Angelelli, 2004), même si ceux-ci se sentent souvent en porte à faux entre la façon dont ils souhaiteraient contribuer à l'interaction et ce qu'on leur demande de faire (Wamba, Leanza et Changkakoti, 2006).

Cette tension entre la recherche d'empathie et le recours à un tiers pour accéder à la parole des répondants met en évidence la nécessité de modèles complexes pour rendre compte des processus de confiance et d'empathie dans une interaction. Edwards, Alexander et Temple (2005) distinguent ainsi différents niveaux et types de confiance : la confiance sociale générale (envers les institutions notamment), la confiance personnelle basée sur la familiarité et la proximité et la confiance abstraite, reposant sur le postulat que la personne se comportera avec un certain degré d'expertise, de compétence et de professionnalisme. Faire confiance implique par ailleurs de répondre à l'incertitude par une prise de risque. De même, la vision classique de l'empathie, focalisée sur l'intervenant (chercheur ou thérapeute), ne suffit plus, surtout lorsqu'on la considère dans le cadre d'interactions à trois (Pugh et Vetere, 2009). La notion d'empathie discursive (Sinclair et Monk, 2005), conçue comme une co-construction graduelle entre tous les participants aux interactions conviendrait mieux.

Dans une perspective de recherche compréhensive, où tous les acteurs sont socioculturellement situés (Pestre, 2006), le traducteur intervient lui aussi dans la construction de l'objet de recherche et des récits recueillis. Le tierstraduisant est porteur de culture(s), il est passé par une expérience migratoire ou en est héritier et, comme nous l'observons dans les recherches présentées ici, il a souvent plusieurs identités professionnelles. Même si le mandat est celui de rester le plus technique possible, comment imaginer que la personne du traducteur n'ait aucune influence dans l'interaction? Ce dernier va-t-il en effet se situer plutôt du côté des répondants, cherchant peut-être à donner une bonne image d'eux, ou plutôt du côté du chercheur, se faisant alors parfois porte-parole d'intentions qui n'étaient pas celles de l'enquêteur?

La littérature évoque des effets contradictoires quant à l'impact de la traduction sur les données recueillies. Murray et Wynne (2001), tout en trouvant que les narrations recueillies avec traducteurs sont plus superficielles, relèvent également qu'il n'y a pas de loi lorsqu'il s'agit de faciliter l'expression, parfois c'est la proximité parfois la distance qui libère la parole. Des entretiens menés directement par un collaborateur de recherche dans la langue des répondants peuvent ainsi permettre le recueil de récits plus fouillés, mais selon les thématiques et les personnes, l'effet peut être inverse.

En ce qui nous concerne, nous avons trouvé dans les deux terrains en contexte migratoire (terrains 1 et 2) que, conformément à nos attentes par rapport au mandat donné de référence culturelle, les traductrices ont révélé d'autres données et ont ainsi élargi les pistes d'interprétation possibles. Sans la médiation culturelle de la traductrice (qui passe par la discussion sur les mots eux-mêmes et la réalité qu'ils activent) l'une des chercheures n'aurait ainsi pas pu saisir toute l'importance de la première coupe de cheveux en tant que l'un des rites initiatiques ${ }^{14}$ rythmant dans la religion bouddhiste l'entrée de l'enfant en " humanité » (terrain 1). La question se pose par ailleurs de la limite de l'intervention du tiers-traduisant: une traductrice peut-elle ainsi «prendre le pouvoir " jusqu'à prolonger l'entretien au-delà de la clôture annoncée par la chercheure (situation relevée sur le terrain 1)? Les recherches menées en contexte médical ou psychothérapeutique ont montré qu'un traducteur change de rôles au cours d'un entretien, que ce soit sous l'impulsion de l'intervenant ou de son propre chef (Goguikian Ratcliff et Changkakoti, 2004; Grin, 2003; Jalbert, 1998; Leanza, 2005). Les observations sur nos différents terrains montrent qu'il en va de même dans un contexte de recherche. La question du contrôle est peutêtre à réinscrire dans celle de l'asymétrie des rôles. Fournier (2006) affirme que dans une recherche il faut assumer l'asymétrie des positions sociales, nous proposons d'étendre cette notion à la recherche avec traducteur (en triade). Soit il s'agit d'un entretien mené par deux chercheurs, avec les mêmes prérogatives par rapport à la recherche - l'un d'eux étant un « professionnel bilingue »-, soit il s'agit d'un entretien mené par un chercheur principal et un traducteur, ce dernier pouvant avoir reçu un mandat de co-chercheur, le chercheur principal restant alors le pilote de l'entretien.

\footnotetext{
${ }^{14}$ Hérités de l'hindouisme.
} 
Nous avons expérimenté deux cas de figure sur nos terrains lors d'entretiens en triade: une traductrice fonctionnant par moments comme co-chercheure (terrain 2), avec l'accord de la chercheure principale, et des interprètes communautaires faisant parfois activement bénéficier l'entretien de leurs connaissances personnelles de la culture des répondants (terrain 1), la chercheure gardant dans les deux cas les commandes de l'entretien. II existe toutefois une zone de flou entre le mandat donné aux traducteurs et la façon dont ceux-ci l'interprètent, ce qui peut conduire à des sentiments d'impuissance et de dépossession comme lorsque la traductrice prend en quelque sorte le contrôle du déroulement de l'interaction en prolongeant de son propre chef l'entretien. Plus le mandat est implicite et plus le risque de frustrations de part et d'autre augmente.

Nos terrains nous présentent un troisième cas de figure: celui de collaboratrices de recherche de même langue, voire de même communauté, que celles auprès desquelles l'enquête est menée (terrains 3 et 4). Certains auteurs (Murray et Wynne, 2001) mentionnent l'intérêt de travailler avec des traducteurs ayant un profil similaire à celui des interviewés, dans le but notamment de réduire l'asymétrie mais d'autres, comme Bernhardt (2010), donnent des exemples contraires où la proximité, dans le sens d'une adhésion totale aux valeurs de la communauté, va jusqu'à empêcher le recueil de données. À Bénarès (terrain 3), cette proximité des collaboratrices de recherche avec le terrain a interféré dans certains cas avec le recueil de données (en particulier pour l'une d'entre elles, hindouiste stricte proche de l'esprit de l'école sanskrite des filles). Une épreuve spatiale a ainsi dû être supprimée car la façon dont la chercheure avait donné la consigne a modifié les stratégies des jeunes filles observées, qui ont eu, de ce fait, toutes les peines du monde à résoudre la tâche. L'objectif de l'épreuve était d'observer l'impact du langage utilisé sur les stratégies métacognitives mises en œuvre dans la tâche, afin de déterminer si celles-ci se référaient ou non à la tradition culturelle, tandis que celui poursuivi, consciemment ou non, par la collaboratrice de recherche était d'assurer l'utilisation des références culturelles sanskrites spécifiques, afin de donner une bonne image de cette forme particulière de scolarisation. Le contrôle des transcriptions a aussi montré que, lors des entretiens, elle a constamment interprété à sa manière (politiquement correcte) les propos des enseignantes de cette école. Ainsi, elle a sciemment minimisé le fait que les filles de cette école s'entraînaient avec des armes réelles.

\section{Conclusion}

La multiplicité des facteurs en jeu lorsqu'on se situe dans une perspective sensible montre que dans nos quatre terrains, y compris celui qui pourrait paraître intraculturel (terrain 4), la recherche passe bien par le prisme interculturel de la traduction pour reprendre les mots de Ladmiral (1998). De ce prisme font partie les appartenances socioculturelles, les contextes de vie et d'observation des différents acteurs, les identités personnelles et professionnelles, les rapports de force qui s'incarnent dans ces acteurs, l'histoire qui les traverse. On peut postuler que toute situation de recherche interculturelle au sens large, même lorsque le chercheur est issu de la communauté étudiée, comporte une asymétrie due à la nécessaire distance de la position de chercheur. L'asymétrie pose problème et interfère avec les aspects sensibles lorsqu'elle reste invisible, inconsciente, négligée, voire niée. Pour filer la métaphore du prisme, on pourrait dire que pour pouvoir analyser les composantes de la communication au sein de l'interaction de recherche, il faut pouvoir repérer les indices de réfraction. Cela implique une approche réflexive (Temple et Edwards, 2002), où le chercheur s'interroge sur son propre positionnement, son contre-transfert (Devereux, 1980; Giami, 2001) sur la thématique de recherche et les personnes avec qui il interagit, où le chercheur questionne les relations de pouvoir entre participants à la recherche, traducteurs inclus, où il ne les "escamote pas de la scène du terrain » (Marcus, 2002) et décrit donc les conditions de recueil des données et le contexte socioculturel, politique et historique. L'analyse du contre-transfert du chercheur travaillant en équipe peut s'effectuer lors de réunions où une attention explicite est donnée à ces processus, comme le rapportent Bernhard (2010) et Giami (2001).

Le malentendu (frottements dans la relation, compréhensions divergentes, incompréhensions) constitue l'élément qui permet par excellence de repérer les indices de réfraction : il donne à voir les divergences de vue, il révèle d'où l'on parle, il oblige à sortir de l'implicite. Sans le malentendu on risque de se trouver dans une illusion de «bien entendu ». Pour ce faire, les malentendus doivent être repérés et explicités, par exemple lors d'une vérification de la traduction. La réflexivité ne s'exerce pas que dans l'après-coup, elle devrait s'effectuer aussi durant l'interaction, en parlant des mots et des réalités qu'ils recouvrent, en explicitant certains choix de langage. Cette nécessité 
d'explicitation avant, pendant et après les entretiens est la recommandation à laquelle aboutissent certains auteurs ayant abordé la problématique de la recherche avec traduction (Bernhard, 2010; Chen, 2011; Murray et Wynne, 2001; Wadensjö, 1998). C'est à cette condition que le flou devient une zone d'intercompréhension et le prisme de la traduction une véritable ressource. Cela demande au chercheur une certaine humilité, celle exigée par la rencontre avec l'autre (La Cecla, 2002), où l'on accepte de ne pas savoir. C'est à ce prix également que l'on peut rester sensible en situation d'asymétrie de langues et de cultures.

Il se pose enfin la question de l'interprétation des données et de leur mise en forme, qui rejoint celle de l'anthropologie comme traduction de cultures. Comment produit-on à partir de la parole des gens et de l'observation des personnes en contexte un récit, texte écrit destiné à la communauté scientifique, qui restitue pour des lecteurs absents de la scène à la fois l'épaisseur du vécu qui a entouré le recueil de données et la pensée qui en est abstraite?

Le chercheur peut être piégé par la recherche d'authenticité, en s'abritant derrière la parole des répondants dans les approches compréhensives, alors que le choix de tel ou tel extrait est loin d'être neutre, ou en tentant d'évacuer du terrain anthropologique de la tradition orale le fait que "les sujets traditionnels produisent euxmêmes des formes de discours et d'auto-représentation systématiques destinés à un monde de représentation contextualisé produit entre autres par les médias, les États, les entreprises et les ONG » (Marcus, 2002). À ce propos, Gonzalez y Gonzalez et Lincoln (2006) proposent de "décoloniser la recherche qualitative » en donnant une place à tous les acteurs de la recherche à toutes les étapes de la recherche, et en se montrant créatif dans la façon de rendre compte des résultats. Cette créativité peut prendre la forme de comptes-rendus plurilingues, de mises en forme non conventionnelles littéraires et artistiques associées aux textes scientifiques classiques. L'explicitation du rôle, de la place et des caractéristiques de chacun des acteurs ne peut qu'augmenter, nous semble-t-il, la validité des résultats obtenus et c'est donc au développement d'une sensibilité accrue des chercheurs aux asymétries présentes dans les interactions verbales que cet article souhaite contribuer.

\section{Références bibliographiques}

Angelelli, C. (2004). Medical Interpreting and Cross-cultural Communication. Cambridge : Cambridge University Press.

Apostolou, F. (2009). Mediation, manipulation, empowerment. Celebrating the complexity of the interpreter's role. Interpreting, 11(1), 1-19.

Benveniste, E. (1966). Problèmes de linguistique générale. Paris : Gallimard.

Bernhard, J. (2010). Quels sont les éléments essentiels de la recherche valable? Les problèmes de " données » et leur cueillette dans les contextes interculturels. Early childhood Education Publications and Research, 3, 2732. http://digitalcommons.ryerson.ca/ece/27

Billiez, J. (1985). La langue comme marqueur d'identité. Revue Européenne des Migrations Internationales, 2(1), 95105.

Bischoff, A., Loutan, L. et Stalder, H. (2001). Barrières linguistiques et communication dans une policlinique de médecine. VALS-ASLA, Bulletin suisse de linguistique appliquée, 74, 193-207.

Blanchet, P. (2000). La linguistique de terrain. Rennes : Presses Universitaires de Rennes.

Broyon, M.-A. (2004). L'éducation sanskrite à Bénares, enjeu d'une société qui oscille entre traditions et transition. Dans A. Akkari et P. Dasen (dir.), Pédagogies et pédagogues du Sud (p. 231-250). Paris : L'Harmattan.

Buzelin, H. (2004). La traductologie, l'ethnographie et la production des connaissances. Meta : journal des traducteurs / Meta : Translators' Journal, 49(4), 729-746.

Changkakoti, N., Gremion, M. et Hutter, V. (2009). Familles migrantes : une transmission culturelle négociée. Interdialogos, 2, 18-21. 
Chen, S. (2011). Power Relations Between the Researcher and the Researched: An Analysis of Native and Nonnative Ethnographic Interviews. Field Methods, 23, 119-135.

Cohen-Emerique, M. (2011). Pour une approche interculturelle en travail social, Théories et pratiques. Rennes : Presses de l’EHESP.

Dasen, P. et Akkari, A. (2008). Educational theories and practices from the majority world. New Delhi : Sage.

Deprez, C. (2002). La langue comme "épreuve » dans les récits de migration. VALS-ASLA, Bulletin suisse de linguistique appliquée, 76, 39-52.

Devereux, G. (1980). De l'angoisse à la méthode dans les sciences du comportement. Paris : Flammarion.

Edwards, R., Alexander, C. et Temple, B. (2006). Interpreting Trust : Abstract and Personal Trust for People Who Need Interpreters to Access Services. Sociological Research Online, 11, 1. http://www.socresonline.org.uk/11/1/edwards.html [consult. le 29 déc. 2011]

Fassin, D. (1999). L'anthropologie entre engagement et distanciation : essai de sociologie des recherches en sciences sociales sur le sida en Afrique. Dans C. Becker, J.P Dozon, Ch. Obbo et M. Touré (dir.), Vivre et penser le sida en Afrique (p. 41-66). Paris : Karthala.

Favret-Saada, J. (1977). Les mots, la mort, les sorts. Sorcellerie dans le bocage. Paris : Gallimard.

Favret-Saada, J. (2009). Désorceler. Paris : L'Olivier.

Fleury, F. et Fierro, I. (2009). La médiation linguistique et culturelle, une nécessité? Évolution et construction du rôle d'interprète communautaire. Lausanne : Appartenances. http://www.appartenances.ch/Assises/ interpretariat/FLEURY_F_FIERRO_I_Mediation_linguistique_culturelle.pdf

Fournier, P. (2006). Le sexe et l'âge de l'ethnographe : éclairants pour l'enquêté, contraignants pour l'enquêteur. Ethnographiques.org, 11. http://www.ethnographiques.org/2006/ Fournier [consult. le 3 janv. 2012]

Gajardo, A. (2009). Qui de la culture ou de la loi fait l'ethnie? Esquisse de réflexion en cours sur le processus de (re)connaissance légale des Diaguita. Tsantsa. Revue de la Société Suisse d'ethnologie, 14.

Giami, A. (2001). Counter-transference in social research: beyond George Devereux. Papers in Social Research Methods - Qualitative Series, 7. http://www.ethnopsychiatrie.net/giami.htm

Goguikian Ratcliff, B. et Changkakoti, N. (2004). Le rôle de l'interprète dans la construction de l'interculturalité dans un entretien ethnopsychiatrique. L'autre. Cliniques, cultures et sociétés, 5(2), 255-264.

Gonzalez y Gonzalez, E. et Lincoln, Y. (2006) Decolonizing qualitative research: non-traditional reporting forms in the academy. FQS. Forum: Qualitative Social Research, 7(4),1. http://www.qualitativeresearch.net/index.php/fqs/article/view/162

Graber, M. et Girod, I. (2001). Communication entre soignants et patients migrants dans différents services hospitaliers [en ligne]. Dans Actes du $8^{e}$ congrès international de l'Association pour la Recherche InterCulturelle (ARIC) [en ligne]. http://www.unifr.ch/ipg/ARIC/8eCongres/DocsOrig/GraberM\%20GirodI.pdf

Grin, C. (2003). Retour à la pratique. Dans P. Guex et P. Singy (dir.), Quand la médecine à besoin d'interprètes (p. 141-163). Genève : Médecine et Hygiène.

Gumperz, J. (1989). Engager la conversation. Introduction à la sociolinguistique interactionnelle. Paris : Minuit.

Hutter, V., Gremion, M., Borruat, S., Ogay, T., Zharkova Fattore, Y. et Gakuba, T. (2008). Quand les chercheurs font une recherche sur la recherche. Zoom sur les défis scientifiques et professionnels d'une expérience interculturelle. Bulletin de I'ARIC, 46, 10-24.

Jalbert, M. (1998). Travailler avec un interprète en consultation psychiatrique. P.R.I.S.M.E, 8(3), 94-111.

Joss, M. (2008). Bien plus que de l'interprétariat. L'interprétariat communautaire, une base de confiance pour le traitement des patients migrants. Bulletin des médecins suisses / Schweizerische Ärztezeitung / Bollettino dei medici svizzeri, 89(8), 320-322.

Alterstice - Revue Internationale de la Recherche Interculturelle, vol. 2, $n^{\circ} 1$ 
Kaufert, J. et Putsch, R. (1997). Communication through interpreters in healthcare : Ethical dilemmas arising from differences in class, culture, language, and power. Journal of Clinical Ethics, 8(1), 71-87.

La Cecla, F. (2002). Le malentendu. Paris : Balland.

Ladmiral, J.-R. (1998). Le prisme interculturel de la traduction. Palimpsestes, 11, 15-30.

Lavanchy, A. et Gajardo, A. (2008, nov.). Qui fait l'interculturalité au Chili? Réflexions transversales à propos des politiques autochtones chiliennes. Communication au colloque de la Société Suisse d'Ethnologie, Genève.

Leanza, Y. (2011). Exercer la pédiatrie en contexte multiculturel. Une approche complémentariste du rapport institutionnalisé à l'Autre. Genève : Georg.

Leanza, Y. (2005). Roles of community interpreters in pediatrics as seen by interpreters, physicians and researchers. Interpreting, 7(2), 167-192.

Londei, D. (2011). Traduire les savoirs. Berne : Peter Lang.

Malinowki, B. (1935). Coral Gardens and their magic. Londres : George Allen et Unwin.

Marcus, G. (2002). Au-delà de Malinowski et après Writing Culture : à propos du futur de l'anthropologie culturelle et du malaise de l'ethnographie. Ethnographiques.org, 1. http://www.ethnographiques.org/2002/Marcus.html

Métraux, J.-C. et Alvir, S. (1995). L'interprète : traducteur, médiateur culturel ou co-thérapeute. Interdialogos, 2, 22-26.

Métraux, J.-C. (2002). L'interprète, ce nouvel acteur. Cahiers psychiatriques, 29, 115-135.

Mishra, R. et Dasen, P. (2007). The methodological interface of psychology and anthropology. Dans J. Wassmann et K. Stockhaus (dir.), Experiencing new worlds. Oxford : Berghan.

Molina, M. (2001). Communication, migration et santé : souffrances psychiques et communication. Comment dire sa souffrance en situation d'insécurité linguistique et socioculturelle. Dans Actes du $8{ }^{e}$ congrès international de l'Association pour la Recherche InterCulturelle (ARIC) [en ligne]. http://www.unifr.ch/ipg/ARIC/8eCongres/DocsOrig/MolinaME.pdf

Murray, C. et Wynne, J. (2001). Researching community, work and family with an interpreter. Community, Work \& Family, 4(2), 157-171.

Payet, J.-P., Giuliani, F. et Laforgue, D. (2008). La voix des acteurs faibles. De l'indignité à la reconnaissance. Rennes : Presses Universitaires de Rennes.

Perregaux, C., Changkakoti, N., Gremion, M. et. Hutter, V. (2010). Des familles migrantes en recherche de dialogues avec l'école. Dans M. Mc Andrew, M. Milot et A. Triki-Yamani (dir.), L'école et la diversité. Perspectives comparées (p. 195-203). Québec: Presses de l'Université Laval.

Pestre, D. (2006). Introduction aux « Sciences Studies ». Paris : La Découverte.

Phillips, H. (1960). Problems of Translation and Meaning in Field Work. Human Organization, 18(4), $184-192$.

Pirinoli, C. (2004). L'anthropologie palestinienne entre science et politique: I'impossible neutralité. Anthropologie et Sociétés, 28(3), 165-185. http://id.erudit.org/iderudit/011288ar

Pugh, M. et Vetere, A. (2009). Lost in translation : An interpretative phenomenological analysis of mental health professionals' experiences of empathy in clinical work with an interpreter. Psychology and Psychotherapy : Theory, Research and Practice, 82, 305-321.

Searight, H. et Searight, B. (2009). Working with foreign language interpreters : recommendations for psychological practice. Professional Psychology : Research and Practice, 40(5), 444-451.

Simon, S. (1988). Excursions ethnologiques : contextes pour penser les pouvoirs de la traduction. Traduction, terminologie, rédaction, 1(2), 28-36. http://id.erudit.org/iderudit/037015ar [consult. le 5 déc. 2011] 
Sinclair, S. et Monk, G. (2005). Discursive empathy : A new foundation for clinical practice. British Journal of Guidance and Counselling, 33, 337-349.

Sirna, F. (2009). L'enquête biographique : réflexions sur la méthode. Dans A. Aggoun (dir.), Enquêter auprès des migrants. Le chercheur et son terrain (p. 9-28). Paris : L'Harmattan.

Temple, B. et Edwards, R. (2002). Interpreters/Translators and Cross-Language Research : Reflexivity and Border Crossings. International Journal of Qualitative Methods, 1(2), 1-12.

Vatz-Laaroussi, M. (2003). Les enjeux méthodologiques de la recherche interculturelle : entre l'histoire, la médiation et l'engagement. Bulletin de I'ARIC, 39, 77-83.

Vulbeau, A. (2007). L'approche sensible des quartiers « sensibles ». Informations sociales, 141, 8-13.

Wadensjö, C. (1998). Interpreting as interaction. London : Longman.

Wamba, A., Leanza, Y. et Changkakoti, N. (2006, sept.). Getting And Transmitting Knowledge Through Interpreters : Power Dynamics And Cooperation. Communication présentée au colloque ECER (European Conference on Educational Research), Transforming knowledge, Université de Genève.

Weiss, R. et Stucker, R. (1998). Interprétariat et médiation culturelle dans le système de soins. Neuchâtel : Rapport de recherche 11 du Forum suisse pour l'étude des migrations. 